\title{
Construction of a fur null mutant and RNA-sequencing provide deeper global understanding of the Aliivibrio salmonicida Fur regulon
}

\author{
Sunniva Katharina Thode ${ }^{1}$, Cecilie Bækkedal ${ }^{1}$, Jenny Johansson Söderberg ${ }^{1}$, Erik Hjerde ${ }^{1}$, Hilde Hansen ${ }^{1}$, \\ Peik Haugen ${ }^{\text {Corresp. } 1}$ \\ ${ }^{1}$ Department of Chemistry and The Norwegian Structural Biology Centre, Faculty of Science and Technology, UiT The Arctic University of Norway, Tromsø, \\ Norway \\ Corresponding Author: Peik Haugen \\ Email address: peik.haugen@uit.no
}

Background. The ferric uptake regulator (Fur) is a transcription factor and the main regulator of iron acquisition in prokaryotes. When bound to ferric iron, Fur recognizes its DNA binding site and generally executes its function by repressing transcription of its target genes. Due to its importance in virulence, the Fur regulon is well studied for several model bacteria. In our previous work, we used computational predictions and microarray to gain insights into Fur-regulation in Aliivibrio salmonicida, and have identified a number of genes and operons that appear to be under direct control of Fur. To provide a more accurate and deeper global understanding of the biological role of Fur we have now generated an A. salmonicida fur knock-out strain and used RNA-sequencing to compare gene expression between the wild-type and fur null mutant strains.

Results. An A. salmonicida fur null mutant strain was constructed. Biological assays demonstrate that deletion of fur results in loss of fitness, with reduced growth rates, and reduced abilities to withstand lowiron conditions, and oxidative stress. When comparing expression levels in the wild-type and the fur null mutant we retrieved 296 differentially expressed genes distributed among 18 of 21 functional classes of genes. A gene cluster encoding biosynthesis of the siderophore bisucaberin represented the highest upregulated genes in the fur null mutant. Other highly up-regulated genes all encoded proteins important for iron acquisition. Potential targets for the RyhB sRNA was predicted from the list of down-regulated genes, and significant complementarities were found between RyhB and mRNAs of the fur, sodB, cysN and VSAL_10422 genes. Other SRNAs with potential functions in iron homeostasis were identified.

Conclusion. The present work provides by far the most comprehensive and deepest understanding of the Fur regulon in A. salmonicida to date. Our data also contribute to a better understanding of how Fur plays a key role in iron homeostasis in bacteria in general, and help to show how Fur orchestrates iron uptake when iron levels are extremely low. 
1 Construction of a fur null mutant and RNA-sequencing provide deeper global

2 understanding of the Aliivibrio salmonicida Fur regulon

3

4 Sunniva Katharina Thode ${ }^{1}$, Cecilie Bækkedal ${ }^{1}$, Jenny Johansson Söderberg ${ }^{1}$, Erik Hjerde ${ }^{1}$, Hilde

5 Hansen $^{1}$ and Peik Haugen ${ }^{1 *}$

$6{ }^{1}$ Department of Chemistry and The Norwegian Structural Biology Centre, Faculty of Science and

7 Technology, UiT - The Arctic University of Norway

$8 \quad *$ Corresponding author: Peik Haugen

9 Email address: peik.haugen@uit.no

10

11

12

13

14

15

16

17

18

19

20

21

22 


\section{Abstract}

24

Background. The ferric uptake regulator (Fur) is a transcription factor and the main regulator of iron acquisition in prokaryotes. When bound to ferric iron, Fur recognizes its DNA binding site and generally executes its function by repressing transcription of its target genes. Due to its importance in virulence, the Fur regulon is well studied for several model bacteria.

In our previous work, we used computational predictions and microarray to gain insights into Fur-regulation in Aliivibrio salmonicida, and have identified a number of genes and operons that appear to be under direct control of Fur. To provide a more accurate and deeper global understanding of the biological role of Fur we have now generated an A. salmonicida fur knockout strain and used RNA-sequencing to compare gene expression between the wild-type and fur null mutant strains.

Results. An A. salmonicida fur null mutant strain was constructed. Biological assays demonstrate that deletion of fur results in loss of fitness, with reduced growth rates, and reduced abilities to withstand low-iron conditions, and oxidative stress. When comparing expression levels in the wild-type and the fur null mutant we retrieved 296 differentially expressed genes distributed among 18 of 21 functional classes of genes. A gene cluster encoding biosynthesis of the siderophore bisucaberin represented the highest up-regulated genes in the fur null mutant. Other highly up-regulated genes all encoded proteins important for iron acquisition. Potential targets for the RyhB sRNA was predicted from the list of down-regulated genes, and significant complementarities were found between RyhB and mRNAs of the fur, $\operatorname{sodB}, \operatorname{cys} N$ and VSAL_I0422 genes. Other sRNAs with potential functions in iron homeostasis were identified.

Conclusion. The present work provides by far the most comprehensive and deepest understanding of the Fur regulon in A. salmonicida to date. Our data also contribute to a better understanding of how Fur plays a key role in iron homeostasis in bacteria in general, and help to show how Fur orchestrates iron uptake when iron levels are extremely low. 
52

53

54

55

56

57

58

\section{Introduction}

The ferric uptake regulator, Fur, represents the main regulator of iron levels in prokaryotic microorganisms (reviewed by Fillat 2014). In addition to regulating iron acquisition genes, Fur also regulates genes involved in e.g., the TCA cycle, DNA metabolism, energy metabolism, redox-stress resistance, chemotaxis, swarming, metabolic pathways, toxin production and other virulence factors, and is therefore considered as a so-called master regulator (Escolar et al. 1999; Hantke 2001; McHugh et al. 2003; Mey et al. 2005a; Pajuelo et al. 2016). Transcriptomic studies on fur null mutants of Vibrio cholerae (Mey et al. 2005a) and Vibrio vulnificus (Pajuelo et al. 2016) have shown that Fur represses expression of siderophore biosynthesis and transport genes, heme transport and utilization genes, ferric and ferrous iron transport genes, stress response and biofilm genes, amongst others. The same studies also showed that Fur activates genes involved in stress responses, chemotaxis, motility and toxin production. In Escherichia coli K-12, Fur directly regulates 131 genes including those of seven other master regulators, i.e., flhD, flhC, felc, soxS, ryhB, rpoS and purR (McHugh et al. 2003), which subsequently can result in regulation of 3158 genes in total (incl. direct and indirect effects), according to EcoCyc (Keseler et al. 2013). This huge number of genes translates to $>70 \%$ of the total number of genes in E. coli K-12 (which is 4318 according to EcoCyc), and illustrates the central role of Fur in cellular processes far beyond iron homeostasis.

The 3D-structure of Fur from Pseudomonas aeruginosa, E. coli, V. cholerae, Helicobacter pyroli and Campylobacter jejuni is known (Butcher et al. 2012; Dian et al. 2011; Pecqueur et al. 2006; Pohl et al. 2003; Sheikh \& Taylor 2009). These structures show that Fur mainly acts as a homodimer in both apo and holo forms, where at least two zinc ligands per monomer stabilize the dimer (Fillat 2014). The iron binding sites are located in a DNA binding domain of each monomer. Here, iron binding causes conformational changes that enable Fur to bind to its DNA target (known as the Fur-box) (Fillat 2014). Although several different Fur-box motifs have been proposed over the years, the current literature have converged on a $19 \mathrm{bp}$ palindromic sequence centered around a non-conserved position (Ahmad et al. 2009; Baichoo \& Helmann 2002; Davies et al. 2011; De Lorenzo et al. 1988; Escolar et al. 1998). Once bound to its DNA target, Fur mainly acts as a repressive regulator by blocking the transcription of downstream genes. 
82

83

84

85

86

87

An apparent gene activating effect by Fur was observed during early investigations of the Fur regulon and was proposed to be due to post-transcriptional regulation (Hantke 2001). This effect was later discovered to originate from negative regulation by Fur of a gene encoding the small regulatory RNA (sRNA) named RyhB (Masse et al. 2003; Massé \& Gottesman 2002; Masse et al. 2005). The RyhB sRNA is responsible for destabilizing mRNAs of its target genes, and repression of $r y h B$ by holo-Fur was therefore interpreted as activation by Fur. RyhB typically targets mRNAs encoding iron-using or iron-binding proteins as a way of preserving the iron levels in the cell at low iron conditions (Davis et al. 2005; Masse et al. 2005; Murphy \& Payne 2007). In E. coli RyhB directly targets 28 mRNAs (see EcoCyc.org). Examples of targets include mRNAs of bfr, cysE, $\operatorname{sod} A B, f u m A, \operatorname{sucBCD}$, icsRSUA, and $s d h A B C D$ (Massé \& Gottesman 2002). In $V$. cholerae RyhB targets mRNAs of $s o d B, s d h C$, gltB1 and fumA. In contrast to E. coli, mRNAs of the iron storage genes like $b f r$ and $f t n$ are not regulated by the $V$. cholerae RyhB (Davis et al. 2005).

The aim of this study was to investigate the Fur regulon in A. salmonicida, the causative agent of cold-water vibriosis in Atlantic salmon (Salmo salar), rainbow trout (Oncorhynchus mykiss) and Atlantic cod (Gadus morhua) at sea-water temperatures below $10^{\circ} \mathrm{C}$ (Colquhoun \& Sorum 2001; Enger et al. 1991). In a previous study we identified a Vibrionaceae-specific Furbox consensus as 5'-AATGANAATNATTNTCATT-3', and used computational methods to predict Fur-regulated genes and operons in four Vibrionaceae genomes, including $A$. salmonicida (Ahmad et al. 2009). Fur-binding motifs were associated with 60 single genes and 20 operons ( 89 genes in total). Later we used molecular dynamics (MD) simulations and binding free energy calculations to gain more insights into the interactions between $A$. salmonicida Fur (asFur) and proposed Fur-binding sites (Pedersen et al. 2010). Here, Fur-binding to promoters was dependent on the number of Fur-boxes, and the predicted "strengths" (i.e., calculated similarity to Fur-box consensus) of the individual Fur-boxes. Finally, we studied Fur-regulation in A. salmonicida using iron-depletion experiments in combination with custom whole-genome microarray chips (Ahmad et al. 2012; Thode et al. 2015). Thirty-two genes were found to be significantly up-regulated 15 min after exposure to low-iron conditions (suggesting Furregulation), and interestingly, the $b i b A B C$ genes encoding the producing proteins for the siderophore bisucaberin were identified as being most highly up-regulated (Thode et al. 2015). We have now constructed an A. salmonicida fur null mutant and used Illumina based RNA- 
113 sequencing (RNA-seq) to compare the transcriptomes of the wild-type strain and the fur null 114 mutant. Overall, we find that the RNA-seq data overlap remarkably well with our previous

115 findings when using microarray. However, we also show that high-throughput RNA-sequencing 116 provide us with a much more accurate and fine-grained global understanding of the Fur regulon 117 in A. salmonicida, compared to what we knew from our previous microarray work.

Materials and methods

Bacterial strains, culture conditions, and sampling for RNA sequencing

121

122

123

124

125

126

127

128

129

130

131

132

133

134

135

136

137

138

139

140

A. salmonicida LFI1238 (Hjerde et al. 2008) was used as parental strain for the construction of the $A$. salmonicida fur null mutant (see below for details). Parental and mutant strains were cultured in LB medium [Luria-Bertani broth Miller, Difco (later corrected to Lysogeny Broth (Bertani 2004))] containing 2.5\% $\mathrm{NaCl}$ at $12^{\circ} \mathrm{C}$ and $200 \mathrm{rpm}$. For E. coli strain $\mathrm{S} 17-1$ the growth conditions were $37^{\circ} \mathrm{C}$ and $200 \mathrm{rpm}$ in $\mathrm{LB}$ medium with $1 \% \mathrm{NaCl}$. The suicide plasmid pDM4 (Milton et al. 1996) was propagated in E. coli $\mathrm{S} 17-1$ cells. For selection of E. coli $\mathrm{S} 17-1$ transformants and A. salmonicida transconjugants, $25 \mu \mathrm{g}$ or $2 \mu \mathrm{g}$ of chloramphenicol/ml was added to the medium, respectively.

For biological characterizations (see below for details) and RNA sequencing sampling, $A$. salmonicida LFI1238 and fur null mutant strains were cultured in LB medium with $1 \% \mathrm{NaCl}$ at $8^{\circ} \mathrm{C}$ and $200 \mathrm{rpm}$. For RNA sequencing, three biological replicates of A. salmonicida LFI1238 and A.salmonicida fur null mutant were grown to mid log growth phase, i.e., at optical density $(600 \mathrm{~nm})$ of approximately 0.5 . Ten $\mathrm{mL}$ samples were harvested, spun down and the cell pellets were then stored at $-80^{\circ} \mathrm{C}$ for later processing.

\section{Construction of an A. salmonicida fur null mutant}

The A. salmonicida fur null mutant was constructed using the suicidal plasmid pDM4 (a map of pDM4 can be found at https:/www.google.com/patents/EP1425037B1?cl=en) and allelic exchange, as described by others (Milton et al. 1996). First we constructed the plasmid $\mathrm{pDM} 4 \Delta f u r$, consisting of merged flanking regions of the fur gene. The upstream flanking region 
141 of the fur gene was amplified by PCR using primers FurA forward (5'-

142 CTACTCGAGATATTTATTTCCCTTTAATTC-3') and FurB reverse (5'-

143 CACGTAAACTAAATATGACTTTTCCTGTATTGG-3'). For amplification of the downstream

144 flanking region, primers FurC forward (5'-TATTTAGTTTACGTGCATAAAAAA-3') and FurD 145 reverse (5'-CCCACTAGTATAACAAAGACTCTACTCCAG-3') were used. The resulting 146 upstream and downstream PCR products were fused together using an overlap PCR, cut with 147 restriction enzymes XhoI and SpeI, and ligated into the corresponding sites of pDM4. The 148 resulting pDM4 $\Delta$ fur construct was transformed into E.coli S17-1 and used as donor cells in 149 conjugation experiments with A. salmonicida as described elsewhere (Bjelland et al. 2012). 150 Briefly, E. coli S-17 transformed with pDM4 4 fur was cultivated to mid-log phase and $A$. 151 salmonicida LFI1238 to stationary phase before they were harvested, centrifuged, and washed 152 with $\mathrm{LB}$ containing $1 \% \mathrm{NaCl}$. Donor and recipient strains were resuspended and spottet on LB 153 agar containing $1 \% \mathrm{NaCl}$ and incubated at room temperature for 6 hours to stimulate 154 conjugation, then at $12^{\circ} \mathrm{C}$ for 15 hours to provide better growth conditions for $\mathrm{A}$. salmonicida. 155 Spotted cells were suspended in $\mathrm{LB}$ containing $2.5 \% \mathrm{NaCl}$ and incubated at $12^{\circ} \mathrm{C}$ with $200 \mathrm{rpm}$ 156 for 24 hours. Next, cultures were spread on LB agar containing $2.5 \% \mathrm{NaCl}$ and $2 \mu \mathrm{g} / \mathrm{ml} \mathrm{CAM}$ 157 and incubated at $12^{\circ} \mathrm{C}$ for four days. Potential transconjugants were verified using PCR.

158 Transconjugants were spread on LB agar containing 5\% sucrose to promote allelic exchange. 159 Disposition of pDM4 was verified using a CAM sensitivity test and A. salmonicida fur null 160 mutant was verified using PCR (see Figure S1A) and DNA sequencing (see Figure S1B) with 161 primers FurE (5'-ATTGGGTACGATTCGCATTC-3') and FurF(5'TTCACAGTGCCAAACTCTGC-3').

For RNA-seq, total RNA was purified from cell pellets using the Masterpure complete DNA \& RNA purification kit (Epicentre) following the manufacturer's protocol, followed by an additional DNA removal step using the DNA-free kit (Applied Biosystems). DNase-treated total RNA was subsequently purified using the RNA cleanup RNeasy MinElute kit (Quiagen). The quality of total RNA preps was determined using a Bioanalyzer and a Prokaryote Total RNA Pico Chip (Agilent Technologies). Finally, ribosomal (r) RNA was removed from each sample 
171 (5 $\mu \mathrm{g}$ total RNA) using the Ribo-Zero rRNA Removal Kit (bacteria) (Epicentre) according to the 172 manufacturer's instructions. rRNA-depleted RNA samples were ethanol precipitated (to recover small RNAs), and analyzed on a Bioanalyzer using mRNA Pico Chips (Agilent Technologies).

RNA-sequencing and data analysis

176

177

178

179

180

181

182

183

184

185

186

187

188

189

190

191

192

193

194

195

196

197

198

RNA-seq libraries were generated from purified rRNA-depleted RNA samples using the strandspecific TruSeq stranded mRNA library prep kit (Illumina), and sequenced at the Norwegian Sequencing Centre using the Illumina NextSeq 500 with mid output reagents with a read length of $75 \mathrm{bp}$ and paired end reads. Details on the RNA-seq data is provided in Table S1. The reads were quality checked using FastQC. Further analysis of the RNA-Seq data was performed using a Galaxy pipeline consisting of EDGE-pro v1.0.1 (Estimated Degree of Gene Expression in Prokaryotes) and DESeq. EDGE-pro was used to align the reads to the A. salmonicida LFI1238 genome (Hjerde et al. 2008), and to estimate gene expression. Differences in gene expression between wild-type and fur null mutant were determined using DESeq. $\log _{2}$ fold changes of the genes were recalculated to $\times$ differential expression values (i.e., $\Delta f u r / w t)$ and genes were defined as significantly differentially expressed based on a $p$-value $\leq 0.05$ and differentially expression values of $\Delta f u r / \mathrm{wt} \geq 2 \times$ and $\leq-2 \times$.

\section{sRNA and mRNA target predictions}

The Rockhopper software (McClure et al. 2013) was used to identify sRNA from the RNA-seq data. Input files in the analysis were fastq files from the RNA-seq data, a protein coding gene position file (.ptt), a non-coding RNA position file (.rnt), and finally genome files from $A$. salmonicida LFI1238 [NC_011312.1 (Chr I), NC_011313.1 (ChrII), NC_011311.1 (pVSAL840), NC_011314.1 (pVSAL320), NC_011315.1 (pVSAL54) and NC_011316.1 (pVSAL43)]. sRNAs identified by Rockhopper were visualized in Artemis and manually curated based on a set of criteria. To be accepted as a potential sRNA, its gene should be (i) located in an intergenic region, (ii) between 30-350 nt in length, (iii) located $30 \mathrm{nt}$ or more from the nearest CDS if on the same strand, and $10 \mathrm{nt}$ if on the complementary strand (based on the method of Toffano- 
199 Nioche et. al. (2012)). RNAs fulfilling the criteria described above were further examined for 200 presence of small open reading frames (sORF) using a method adopted from Meulen et al.

201 (2016), since there is an increasing awareness of their presence in bacterial genomes although 202 their significance is not fully understood (Hobbs et al. 2011). Finally, EDGE-pro and DESeq was 203 used to estimate differential gene expression levels for the sRNAs/sORFs.

204

205

206

207

208

209

210

211

212

213

TargetRNA2 and IntaRNA were used to identify potential sRNAs targets (Busch et al. 2008; Kery et al. 2014). Using sRNA sequences as queries, the programs searches for complementary regions in 5' regions of mRNAs. Only targets predicted by both programs were accepted. We also searched for mRNA targets for up-regulated sRNAs (ten sRNAs with folds $\Delta f u r /$ wt $\geq 2 \times$ in the RNA-seq dataset), including RyhB, among the 34 most down-regulated genes in our RNA-seq data set. This was done to identify sRNAs with critical roles in iron homeostasis ( similar to RyhB). In addition, we predicted binding between RyhB and its verified targets ( $\operatorname{sodB}, \mathrm{glt} B, \operatorname{sdhC}$ and $f u m A)$ verified experimentally in E. coli and $V$. cholerae.

Nucleotide sequences of RyhB targets were extracted from European Nucleotide Archive (ENA). The nucleotide sequences were aligned with corresponding sequences in A. salmonicida and examined using Jalview (Waterhouse et al. 2009).

\section{Biological characterization of A. salmonicida fur null mutant}

A. salmonicida LFI1238 wt and fur null mutant ( $\Delta$ fur) were cultured in LB (Difco) at $8^{\circ} \mathrm{C}$ and $200 \mathrm{rpm}$ in all experiments. Growth of cultures was monitored with optical density measured at $600 \mathrm{~nm}$. To determine growth effects of fur null mutation, four replicates of $A$. salmonicida LF1238 wt and $\Delta f u r$ were cultured from lag phase until stationary phase. To determine the ability of the fur null mutant to withstand low iron conditions, wt and $\Delta$ fur cultures were first grown to $\mathrm{OD}_{600 \mathrm{~nm}}$ of 0.38 and 0.33 (mid log phase), respectively. The cultures were then split into five separate flasks. One culture was kept as control whereas $25-500 \mu \mathrm{M}$ of the iron chelator 2,2'dipyridyl was added to the remaining cultures. To determine the ability of the fur null mutant to withstand oxidative conditions, wt and $\Delta$ fur cultures were first grown $\mathrm{OD}_{600 \mathrm{~nm}}$ of 0.4 and 0.35 (mid log phase), respectively. The cultures were then split into five separate flasks. One culture 
227 was kept as control whereas $50-1000 \mu \mathrm{M}$ of hydrogen peroxide was added to the remaining

228 cultures. Growth was monitored for approximately 40 hours.

\section{Results and discussion}

Construction and basic characterization of an A. salmonicida fur null mutant

232

233

234

235

236

237

238

239

240

241

242

243

244

245

246

247

248

249

250

251

252

253

254

To better understand the Fur regulon in A. salmonicida, a fur null mutant was constructed using the genetic system described by Milton et al. (1996). Briefly, approximately $250 \mathrm{bp}$ of upstream and $250 \mathrm{bp}$ downstream sequences flanking the fur gene were merged and inserted into the pDM4 suicide vector (contains $s a c B R$ ), which was then transformed into E. coli S17-1 cells, and finally conjugated into A. salmonicida LFI1238 to trigger recombination and deletion of fur. The fur null mutant was verified by PCR and sequencing.

Basic characterization of the fur null mutant was done to examine the physiological and morphological effects of the fur deletion. Because Fur is a global regulator, we expected the fur null mutant to loose fitness due to loss of control of central cellular processes. For example, loss of Fur is expected to reduce the growth rate, and result in reduced ability to respond to external chemical stress, such as presence of $\mathrm{H}_{2} \mathrm{O}_{2}$ and iron chelators (Becerra et al. 2014; Fillat 2014; Hassett et al. 1996; Touati 2000; Yang et al. 2013). Effects on growth was monitored by comparing the growth rates of the wild-type and the fur null mutant in $\mathrm{LB}$ with $1 \% \mathrm{NaCl}$ at $8^{\circ} \mathrm{C}$ and $200 \mathrm{rpm}$ shaking. The $\mathrm{OD}_{600 \mathrm{~nm}}$ of the starting cultures were set to 0.01 and then monitored until cultures reached stationary phase (typically $\mathrm{OD}_{600 \mathrm{~nm}} 1.2-1.4$ ). The lag phase for the wt and fur null mutant lasted approximately 10 and 35 hours, respectively, and doubling times were approximately 6 and 12 hours during mid log phase (Figure S2A and S2B). To test the ability to respond to chemical stress the fur null mutant and the wild-type strain were exposed to increasing concentrations of hydrogen peroxide $\left(\mathrm{H}_{2} \mathrm{O}_{2}\right)$ and the iron chelator 2,2'-dipyridyl. The minimum inhibitory concentration of $\mathrm{H}_{2} \mathrm{O}_{2}$ on growth for the wild-type and fur null mutant were $500 \mu \mathrm{M}$ and $50 \mu \mathrm{M}$, respectively (Figure S3A and Figure S3B). In a similar experimental setup with 2,2'-dipyridyl the effects were less dramatic (Figure S3C and Figure S3D). The minimum inhibitory of 2,2'-dipyridyl concentrations were similar (approx. $100 \mu \mathrm{M}$ ) for both wild-type 
255 and mutant strain. However, whereas the wild-type strain grows well in the presence of $1 \mathrm{mM}$

2562,2 '-dipyridyl, the fur null mutant cannot grow in the presence of $500 \mu \mathrm{M}$.

257 In summary, deletion of the fur gene results in longer lag phase during growth, longer

258 cell doubling time and reduced ability to respond to oxidative reagents and iron chelators. This is

259 in agreement with results from other $\gamma$-proteobacteria model organisms, e.g., $V$. vulnificus $\Delta$ fur

260 shows higher sensitivity to oxidative stress, reduced fitness and growth (Pajuelo et al. 2016) and

$261 V$. cholerae $\Delta$ fur shows reduction in logarithmic growth (Mey et al. 2005a), and support the

262 validity of the $A$. salmonicida fur mutant.

RNA-sequencing identifies 296 differentially expressed genes in the A. salmonicida fur null mutant

266

267

268

269

270

271

272

273

274

275

276

277

278

279

280

281

282

283

To provide accurate data on the Fur regulon we next compared the transcriptome of the $A$. salmonicida fur null mutant and the wild-type using an RNA-seq approach. RNA samples (from three biological replicates) were prepared from A. salmonicida LFI1238 wild-type and fur null mutant cells grown in $\mathrm{LB}$ containing $1 \% \mathrm{NaCl}$ at $8^{\circ} \mathrm{C}$ to mid $\log$ phase $\left(\mathrm{OD}_{600 \mathrm{~nm}} \approx 0.5\right)$. The given temperature and salt concentration were chosen because $A$. salmonicida is responsible for development of cold-water vibriosis in Atlantic salmon (i.e., at physiological salt conditions) at temperatures below $10^{\circ} \mathrm{C}$ (Bergheim et al. 1990; Colquhoun \& Sorum 2001). RNA samples from biological replicates were subjected separately to paired-end RNA-seq using Illumina NextSeq 500 with $75 \mathrm{bp}$ read length. Sequencing generated an average output of approximately 54 million reads per sample. RNA-seq data was analyzed using a Galaxy pipeline running EDGE-pro v1.0.1 and DESeq. EDGE-pro was used to align reads to the $A$. salmonicida LFI1238 genome, and estimate gene expression. Comparison of gene expression between wild-type and fur null mutant were done using DESeq. Reads originating from rRNA and tRNA genes were excluded from the data analysis. Threshold values for differential expression were set to $\geq 2 \times$ difference (equal to $\log _{2}=1$ ), and with p-value $\leq 0.05$.

Figure 1 shows how a total of 296 differentially expressed genes are distributed among functional gene classes (functional classes adapted from MultiFun (Serres and Riley 2000)). One hundred sixty-two and 134 genes are up-regulated and down-regulated, respectively. The 
284 complete list of the 296 differentially expressed genes are presented in Table S2. All functional

285 classes, except "ribosome constituents", "nucleotide biosynthesis" and "cell division", are 286 represented, and the two classes "cell envelope" and "transport/binding proteins" contain the

287 highest number of genes. Considerable up-regulation of genes from the two latter classes is 288 expected since Fur generally regulates genes as a repressor (Fillat 2014), and loss of Fur is

289

290

291

292

293

294

295

296

297

298

299

300

301

302

303

304

305

306

307

308

309

310

311

312

therefore expected to result in up-regulation (in fur null mutant) of genes involved in iron binding and transport over the membranes. Down-regulated genes are more evenly distributed among 18 of the 21 functional classes, including central processes such as "energy metabolism", "central metabolism", "amino acid biosynthesis" and "cell processes". Although there is no clear pattern, the combined data of up-regulated and down-regulated genes support that asFur is a master regulator with functions similar to that of Fur in E. coli (ecFur) (McHugh et al. 2003).

\section{Chromosomal distribution of differentially expressed genes}

Table 1 and Table 2 summarize details of genes and operons that are up- or down-regulated, Figure 2 shows the chromosomal distribution and positions of the differentially expressed genes, and Figure 3 shows details on RNA-seq reads mapped against the genome for a selection of genes and operons (that will be discussed in more detail below). Previous studies have shown a strong correlation between the distance of genes from oriC (Chr I), and their general transcription level (also known as the gene dosage effect) (Dryselius et al. 2008; Toffano-Nioche et al. 2012). That is, genes located close to oriC are, statistically, more likely to be transcribed at higher levels than genes located further away from oriC, and we were curious to see if asFurrelated genes are found clustered at specific regions of Chr I, perhaps with relevance to their expression levels due to gene dosage.

In our experimental setup the average RPKM value for the upper half of Chr I (i.e., the region closest to oriC) is significantly higher compared to that of the lower half $(660 / 330$ for wild-type and 560/397 for fur null mutant). Gene dosage effects have yet to be demonstrated for Chr II (Dryselius et al. 2008; Toffano-Nioche et al. 2012), which is in agreement with the RPKM values in our experiment (RPKM values are similar for the upper and lower halves of the 
313 chromosome). Differentially expressed genes appear to be relatively evenly distributed on the

314 chromosome, except for some clustering of genes between Chr I pos. 1.85-2.01 Mb. They

315

316

317

318

319

320

321

322

323

324

325

326

327

328

329

330

331

332

333

334

335

336

337

338

339

340

represent a TonB1 system, heme transport and utilization, and cell envelope genes (up-regulated genes), and oxidative stress response, metabolism and sRNAs (down-regulated genes). In other words, there is apparently no clear pattern with respect to asFur-regulated genes and their genomic position. It is interesting to note, however, that the bisucaberin biosynthesis gene cluster and $r y h B$ (encodes the RyhB sRNA) are both located close to oriC. We have previously reported that the bisucaberin biosynthesis system is included in the immediate response to iron limitations in A. salmonicida (Thode et al. 2015), and its genomic location may contribute to the high level of expression and fast response to iron starvation.

\section{asFur regulates iron acquisition systems}

As expected, a high proportion of up-regulated genes (28 of 64) are directly associated with iron metabolism, e.g., siderophore biosynthesis and transport, TonB systems (delivery of energy to iron transport), and heme uptake and utilization. The most up-regulated $(92 \times)$ gene is bibA, which together with the two downstream genes $b i b B C(48 \times$ and $11 \times$ up-regulated in the fur null mutant, respectively) are responsible for producing the siderophore bisucaberin. The overall transcription level for the $b i b A B C$ genes also varies dramatically (see Figure 3A), and follows a trend that more reads map to the first genes of the operons. Therefore, the expression pattern follows the differential expression values for the operon (i.e., $92 \times, 48 \times$ and $11 \times$ ). Interestingly, within the large Vibrionaceae family bibABC are restricted to A. salmonicida and Aliivibrio logei (Kadi et al. 2008; Thode et al. 2015), and are in A. salmonicida (together with a siderophore transport system, bitABCDE) flanked by transposable elements (i.e., a genomic island; see (Hjerde et al. 2008)). Homology search with the BibABC amino acid sequences from $A$. salmonicida, revealed that the close relative Aliivibrio wodanis also possesses the bisucaberin biosynthesis system. The coverage and identity percentage from blastP (with A.salmonicida sequences used as query) were $87 \%$ identity over $100 \%$ coverage for BibA, $90 \%$ identity over 99\% coverage for BibB and 89\% identity over 100\% coverage for BibC. 
Other siderophore receptors and iron-related transport systems that are significantly up-

342 regulated in the fur null mutant include the ferrichrome transport system [VSAL_II0150-0152

$343(6.7-12.5 \times)$ ], the ferrioaxamine B receptor [VSAL_II0909 $(18.8 \times)$ ] and its associated ABC

344 transporters [VSAL_II0907 (5.9×) and II0908 (18.8×)]. A siderophore ferric reductase

345 [VSAL_II0148 $(8 \times)]$ responsible for removing iron from the siderophore, the TonB1 system

346 [VSAL_I1751-1753 (18.8-28.4×)], and finally $h u v B, h u v C$ and $h u v D$ [VSAL_I1754-I1756

$347(5.8-39.7 \times)]$ responsible for heme transport, are up-regulated in the fur null mutant (see Figure

$3483 \mathrm{C}$ for expression details). The heme uptake and utilization gene huvX [VSAL_I1749 (20.2×)]

349 and $p h u W[$ VSAL_I1750 (39.7×)], which encode a putative coproporphyrinogen oxidase

350 believed to be responsible for removing iron from heme, are highly up-regulated in the fur null

351 mutant. The TonB2 system [VSAL_II0110-II0116 (55.8-17.3× up-regulated)] (Figure 3B),

352 iron(III) ABC transporters [VSAL_II0907 (5.9×) and II0908 (11.2×)] and a siderophore receptor

353 gene $\operatorname{des} A$ [VSAL_II0909 $(18.8 \times)$ ] are all highly up-regulated. Interestingly, feo $A B C$

354 (VSAL_I2257-I2259) that encode the ferrous iron transport system, are apparently not strongly

355 regulated by Fur, as only $f e o C$ from this system has an up-regulation $\geq 2 \times$ (i.e., $2.3 \times$ ).

356 In summary, removal of the fur gene results in up-regulation of 28 genes directly

357 associated with iron homeostasis (siderophore biosynthesis, transport and utilization, heme

358 transport and utilization, ABC transporters and TonB1 and TonB2 systems). bibA is by far the

359 most up-regulated $(92 \times)$ gene, whereas the remaining iron-relevant genes are up-regulated $36055-5 \times$.

361

362

asFur regulates several metal transport systems

363 As shown in Figure 1 and Table 1, several transport systems are up-regulated in the fur null mutant. asFur may be involved in the homeostasis of other metals than iron, as multi metal resistance protein genes, a multidrug efflux pump, and nickel and zinc transporter genes are upregulated. In detail; the multi metal resistance genes zntA (VSAL_I2067) and VSAL_II0143 are up-regulated $8.5 \times$ and $5.7 \times$, respectively. The multidrug efflux pump encoded by $v c m D\left(\mathrm{VSAL}_{-}\right.$ I2891) is $8.5 \times$ up-regulated. A large operon (VSAL_II0118-II0125) with annotated nickel and zinc transporters is also up-regulated 4.1-25.7×. Also, the outer membrane protein A gene 
370 (VSAL_I1819), a MFS transporter gene (VSAL_II0149) and potE (VSAL_II1067) are up-

371 regulated $5.9 \times, 5.6 \times$ and $5.0 \times$, respectively.

372

373

Down-regulated genes in asFur null mutant

374 Fur primarily functions as a repressor. The down-regulated genes in our study (i.e., in the fur null

375 mutant) are expected to be positively regulated by asFur in the wild-type, either via the

376 repression of $r y h B$ (or other sRNAs with similar function), which typically destabilizes its

377 mRNA targets (Oglesby-Sherrouse \& Murphy 2013), or by direct stimulation of expression by

378 asFur itself. In this study, we cannot conclusively distinguish between these two possibilities,

379 although we have predicted potential targets of RyhB and other up-regulated sRNAs (see below).

380

Table 2 shows 34 down-regulated genes in the fur null mutant compared to wild-type.

381 Overall, the $\Delta f u r / w t$ values for down-regulated genes are significantly lower than that of up-

382 regulated genes (the strongest down-regulation is $-8.6 \times$, when excluding fur that has been deleted

383 from the genome). In Table 2 we therefore present genes that are $\leq-3 \times$ down-regulated. The

384 majority of the genes are categorized as "motility/chemotaxis" or "metabolism". "Metabolism"

385 genes are involved in different pathways such as amino acid, energy, nucleotide, carbon etc.

386 Moreover, several motility and chemotaxis genes are down-regulated between $-3.5 \times$ and $-6.3 \times$.

387 Of these, four encode flagellin subunits [flaC-flaE (VSAL_I2317- I2319) (Figure 3) and flaF

388 VSAL_I2517)], one encodes a sodium-type polar flagellar protein MotX (VSAL_2771) (Figure

389 3), and two encode methyl-accepting chemotaxis proteins (VSAL_I0799 and VSAL_I2193).

390 Three heat shock proteins encoded by groL1 (VSAL_I0017), groS1 (VSAL_I0018) and $h t p G$

391 (VSAL_I0814) are also down-regulated. Heat shock proteins are involved in protein folding and

392 unfolding, cell cycle control, transport and stress responses amongst others. Transcriptome

393 studies of a $\Delta$ fur mutant in $V$. vulnificus have also shown a down-regulation of heat shock protein

394 genes, chemotaxis protein genes and motility-associated genes (Pajuelo et al. 2016). Two

395 oxidative stress response protein encoding genes, sodB and catA (VSAL_I1858 and

396 VSAL_II0215), are down-regulated (Figure 3). SodB is an iron binding protein and a RyhB

397 target in other organisms, and CatA is a heme-binding protein. 
In summary, differentially down-regulated genes in the $A$. salmonicida fur null mutant

399

400

401

402

403

404

405

406

407

408

409

410

411

412

413

414

415

416

417

418

419

420

421

422

423

424

425

426

427

428

have significantly lower differential expression values than the up-regulated genes possibly due to, in part, secondary regulatory effects rather than direct regulation by Fur. The majority of down-regulated genes have functions in chemotaxis, motility, heat shock and oxidative stress response.

\section{Identification of $S R N A$ s with roles in iron homeostasis}

ncRNAs represent an important part of regulons in bacteria, often controlling critical and early steps in pathways (Gottesman 2005). We therefore set out to explore the presence and function of sRNAs in our RNA-seq dataset. Table 1 and Figure 3D already showed us that $r y h B$ is upregulated $43 \times$ in the fur null mutant, which strongly supports that RyhB in A. salmonicida has a similar role in iron homeostasis as what was established for its homologs in e.g., E. coli (Masse et al. 2005; Seo et al. 2014) and V. cholerae (Davis et al. 2005). Here, RyhB is produced under low-iron conditions and stops production of iron-using and iron-storing proteins, and therefore contributes to a lowered demand for iron (Jacques et al. 2006, Smaldone et al. 2012).

To search for other sRNAs with potential roles in iron homeostasis we re-analyzed the RNA-seq dataset. The rational was that any Fur-regulated sRNA gene are likely candidates to have roles in iron metabolism by targeting specific mRNAs for degradation. One sRNA gene (VSAL_II2005s) that fulfilled this criterion was identified among 252 sRNA genes that we predicted in a previous work (Ahmad et al. 2012). VSAL_II2005s was up-regulated 4×. Furthermore, we analyzed the RNA-seq data using Rockhopper. Rockhopper predicts ncRNAs from RNA-seq data. The sRNAs predicted by Rockhopper were manually curated using the Artemis software. Briefly, to be accepted as a true sRNA, its gene had to be (i) located in an intergenic region, (ii) between 30-350 nt in length, (iii) located $30 \mathrm{nt}$ or more from the nearest CDS if on the same strand, and $10 \mathrm{nt}$ if on the complementary strand. after manual curation, eight of which overlapped or located on the complementary strand of previously predicted sRNAs (Ahmad et al. 2012). These eight sRNAs are VSAL_I4057s, VSAL_I4069s and VSAL_I4164s (overlapping), and VSAL_I4107s, VSAL_I4164s, VSAL_I4189s, VSAL_II2008s and VSAL_II2050s (complementary). Of the remaining nine new 
429

430

431

432

433

434

435

436

437

438

439

440

441

442

443

444

445

446

447

448

449

450

451

452

453

454

455

456

457

458

459

sRNAs identified by Rockhopper and manual curation, six are located on Chr I and three on Chr II (see Figure 4). sRNAs 4 and 7 both contain sORFs, which potentially encode small proteins (see Material and methods) (Hobbs et al. 2011). In general, reads that map to the region predicted by Rockhopper seem to be a sRNA gene. However, for sRNA 8 reads map to a larger region surrounding the region predicted by Rockhopper (see Figure $4 \mathrm{H}$ ). This discrepancy is likely due to that the sRNA is longer than predicted, or alternatively a false positive. The nine new sRNAs were added to the $A$. salmonicida genome annotation using Artemis, and the RNAseq data was re-analyzed for differentially expressed genes using EDGE-pro and DESeq. Two of the sRNAs, i.e., number 1 and 9, were up-regulated $2.2 \times$ and $2.5 \times$ in the fur null mutant, respectively. Homology searches in ENA did not produce significant hits.

In summary, RyhB and a previously predicted sRNA (VSAL_II2005s) were up-regulated in the A. salmonicida fur null mutant. Nine new sRNAs were identified using Rockhopper and manual curation, of which two were differentially expressed (i.e., Figure 4A and Figur4I). Notably, these newly identified sRNAs should be considered as putative until further evidence firmly establishes their presence, e.g., by Northern blot and RACE analyses.

\section{$s R N A$ target predictions}

Next, we used the TargetRNA2 and IntaRNA software to test if the up-regulated sRNAs identified above can explain some of the down-regulated protein-coding genes. The up-regulated sRNAs $r y h B$, VSAL_II2005s and new sRNAs 1 and 9 (see Figure 4A and Figure 4I) were tested for target binding towards the 34 down-regulated genes presented in Table 2. ryhB is upregulated $43.7 \times$, and typically targets mRNA for iron using and iron storage proteins (Davis et al. 2005; Masse et al. 2005; Mey et al. 2005b; Murphy \& Payne 2007; Oglesby-Sherrouse \& Murphy 2013). We expected to find same/similar targets in our dataset. Our results show that RyhB targets seven of the mRNAs listed in Table 2. sodB and fur represent known targets from other organisms (Davis et al. 2005; Masse et al. 2005; Mey et al. 2005b). The other identified

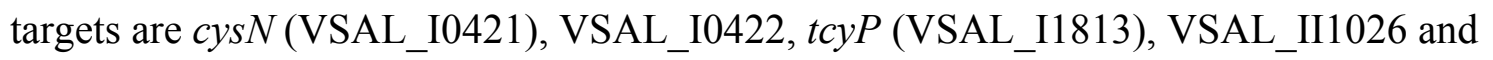
VSAL_I0424. Furthermore, we tested other known targets for complementarity to RyhB.

Matches were found to $g l t B$ and $s d h C$, which were down-regulated $2.1 \times$ and $1.3 \times$, respectively. We therefore consider $g l t B$ as a potential RyhB target in $A$. salmonicida, whereas $s d h C$ is probably not (due to the weak regulation). In E. coli K-12 and Bacillus Subtilis, GltB is an iron- 
460 sulfur binding protein (Miller \& Stadtman 1972; Smaldone et al. 2012). Thus, down-regulation

461 of $g l t B$ is an iron sparing strategy (Jacques et al. 2006, Smaldone et al. 2012).

462 Our target predictions for VSAL_II2005s (which was 4× up-regulated) suggest

463 significant complementarity to $t c y P$ (VSAL_I1813). Interestingly, $t c y P$ was also identified as a

464 RyhB target, which may explain why $t c y P$ has a relative strong down-regulation of $-8.6 \times$ when

465 compared to the other down-regulated genes. No potential targets were identified for sRNAs 1

466 and 9 in Figure 4.

467 In summary, as RyhB appears to have similar regulatory functions as its known homologs

468 from other model organisms, and may account for the down-regulation of seven of the 34 genes

469 in Table 2. We also identified tcyP as a potential target for both RyhB and VSAL_II2005s. No

470 complementarity was found between the newly identified sRNAs 1 and 9 and mRNAs

471 corresponding to the down-regulated genes listed in Table 2.

472

\section{Concluding remarks}

474 We have studied the Fur regulon of $A$. salmonicida using gene knock out technology, and

475 compared the transcriptome of the fur null mutant with its isogenic wild-type using RNA

476 sequencing. Our results show that asFur acts as a master regulator in A. salmonicida affecting

$477 \sim 7 \%$ of the CDSs, when threshold values were set to $2 \times$ differential expression and $p$-value

$478 \leq 0.05$. We also demonstrate that $a s$ Fur acts mainly as a repressor. This conclusion is based on

479 that $\Delta f u r /$ wt differential expression values of up-regulated genes in the fur null mutant are

480 significantly higher than that of down-regulated genes. Furthermore, we demonstrated a strong

481 gene dosage effect for Chr I. This result adds to the growing list of Vibrionaceae bacteria where

482 the transcription level is, statistically, highest for chromosomal regions surrounding oriC $C_{I}$, and

483 weaker for genes located on the opposite end of the chromosome (surrounding $\operatorname{ter} C_{I}$ ). Finally, we

484 identify sRNAs with potential roles in iron homeostasis. The role for RyhB is well established,

485 and in addition, we identified VSAL_II2005s, which was $4 \times$ up-regulated in a fur null mutant,

486 and contains extensive potential for base pairing to the RyhB target $t c y P$ (VSAL_I1813).

Our current data is in good overall agreement with our previous work (Ahmad et al. 2012;

Ahmad et al. 2009; Pedersen et al. 2010; Thode et al. 2015). For example, our current data

overlap with results from our previous works where $A$. salmonicida was subjected to low-iron 
490 conditions and global changes in gene expression was monitored using microarray (Thode et al. 491 2015). Twenty-eight of the 32 genes identified by microarray were $\geq 2 \mathrm{x}$ up-regulated in the fur 492 null mutant. With the latest data we conclude that we today have a more accurate and fine493 grained global understanding of the Fur regulon in A. salmonicida.

\section{Availability of supporting data}

496

497

498

499

500

501

502

503

504

505

506

507

508

509

510

511

512

513

514

RNA sequencing data are accessible in the European Nucleotide Archive (ENA) under accession number PRJEB17700.

\section{Additional files}

Figure S1. Verification of $A$. salmonicida fur null mutant.

Figure S2. Linear (A) and logarithmic (B) growth curves of $A$. salmonicida LFI1238 wt and fur null mutant.

Figure S3. Chemical titration test of $A$. salmonicida LFI1238 wt and fur null mutant.

Table S1. Details on RNA-seq data.

Table S2. Genes that are $\geq 2 \times$ or $\leq-2 \times$ differentially expressed in A. salmonicida fur null mutant compared to wild type.

\section{Abbreviations}

ABC transporter: ATP-binding cassette; Fur: Ferric Uptake Regulator; ecFur: Escherichia coli Fur; asFur: Aliivibrio salmonicida Fur; sRNA: small regulatory RNA; ORF: Open reading frame; mRNA: messenger RNA; TCA: tricarboxylic acid; DNA: Deoxyribonucleic acid; RNA: Ribonucleic acid; bp: base pair; nt: nucleotide; LB: Luria Bertani broth/ Lysogen Broth; tRNA: transfer RNA; rRNA: ribosomal RNA; Chr: Chromosome; MFS transporter: major facilitator 
515 superfamily transporter; h: hours; PCR: Polymerase Chain Reaction; OD: optical density; wt:

516 wild-type; RPKM: reads per kilo base per million mapped reads; RNA-seq: RNA sequencing;

517 rpm: rounds per minute; AS: Aliivibrio salmonicida; sORF: small open reading frame; ncRNA:

518 non-coding RNA; $\Delta$ fur: fur null mutant.

\section{Acknowledgments}

521

522

523

524

525

526

527

528

529

530

531

532

533

534

535

536

537

538

539

540

541

542

543

544

545

546

547

548

549

550

551
The sequencing service was provided by the Norwegian Sequencing Centre (www.sequencing.uio.no), a national technology platform hosted by the University of Oslo and supported by the "Functional Genomics" and "Infrastructure" programs of the Research Council of Norway and the Southeastern Regional Health Authorities.

\section{References}

EcoCyc. Available at http://ecocyc.org/ (accessed 09.22.16).

Google patents. Available at https://www.google.com/patents/EP1425037B1?cl=en (accessed 03.27.16) Ahmad R, Hansen GÅ, Hansen H, Hjerde E, Pedersen HL, Paulsen SM, Nyrud ML, Strauss A, Willassen NP, and Haugen P. 2012. Prediction, Microarray and Northern Blot Analyses Identify New Intergenic Small RNAs in Aliivibrio salmonicida. J Mol Microb Biotech 22:352-360.

Ahmad R, Hjerde E, Hansen G, Haugen P, and Willassen N. 2009. Prediction and experimental testing of ferric uptake regulator regulons in vibrios. J Mol Microbiol Biotechnol 16:159 - 168.

Baichoo N, and Helmann J. 2002. Recognition of DNA by Fur: a reinterpretation of the Fur box consensus sequence. J Bacteriol 184:5826 - 5832.

Becerra G, Merchán F, Blasco R, and Igeño MI. 2014. Characterization of a ferric uptake regulator (Fur)mutant of the cyanotrophic bacterium Pseudomonas pseudoalcaligenes CECT5344. J Biotechnol 190:2-10.

Bergheim A, Kroglund F, Vatne D, and Rosseland B. 1990. Blood plasma parameters in farmed Atlantic salmon (Salmo salar L.) transferred to sea cages at age eight to ten months. Aquaculture 84:159 $-165$.

Bertani G. 2004. Lysogeny at Mid-Twentieth Century: P1, P2, and Other Experimental Systems. J Bacteriol 186:595-600.

Bjelland AM, Sørum H, Tegegne DA, Winther-Larsen HC, Willassen NP, Hansen H. 2012. LitR of Vibrio salmonicida is a salinity-sensitive quorum-sensing regulator of phenotypes involved in host interactions and virulence. Infect Immun 80(5):1681-9.

Busch A, Richter AS, and Backofen R. 2008. IntaRNA: efficient prediction of bacterial sRNA targets incorporating target site accessibility and seed regions. Bioinformatics 24:2849-2856.

Butcher J, Sarvan S, Brunzelle JS, Couture JF, and Stintzi A. 2012. Structure and regulon of Campylobacter jejuni ferric uptake regulator Fur define apo-Fur regulation. Proc Natl Acad Sci U S A 109:1004710052. 
552

553

554

555

556

557

558

559

560

561

562

563

564

565

566

567

568

569

570

571

572

573

574

575

576

577

578

579

580

581

582

583

584

585

586

587

588

589

590

591

592

593

594

595

596

597

598

599

Colquhoun D, and Sorum H. 2001. Temperature dependent siderophore production in Vibrio salmonicida. Microb Pathog 31:213 - 219.

Davies B, Bogard R, and Mekalanos J. 2011. Mapping the regulon of Vibrio cholerae ferric uptake regulator expands its known network of gene regulation. Proc Natl Acad Sci U S A 108:12467 12472.

Davis B, Quinones M, Pratt J, Ding Y, and Waldor M. 2005. Characterization of the small untranslated RNA RyhB and its regulon in Vibrio cholerae. J Bacteriol 187:4005 - 4014.

De Lorenzo V, Giovannini F, Herrero M, and Neilands J. 1988. Metal ion regulation of gene expression: Fur repressor-operator interaction at the promoter region of the aerobactin system of pCoIVK30. J Mol Biol 203:875 - 884.

Dian C, Vitale S, Leonard GA, Bahlawane C, Fauquant C, Leduc D, Muller C, de Reuse H, Michaud-Soret I, and Terradot L. 2011. The structure of the Helicobacter pylori ferric uptake regulator Fur reveals three functional metal binding sites. Mol Microbiol 79:1260-1275.

Dryselius R, Izutsu K, Honda T, and lida T. 2008. Differential replication dynamics for large and small Vibrio chromosomes affect gene dosage, expression and location. BMC Genomics 9:1-16.

Enger O, Husevag B, and Goksoyr J. 1991. Seasonal variations in precense of Vibrio salmonicida and total bacterial counts in Norwegian fish-farm water. Can J Microbiol 37:618 - 623.

Escolar L, Perez-Martin J, and de Lorenzo V. 1998. Binding of the fur (ferric uptake regulator) repressor of Escherichia coli to arrays of the GATAAT sequence. J Mol Biol 283:537-547.

Escolar L, Perez-Martin J, and de Lorenzo V. 1999. Opening the Iron Box: Transcriptional Metalloregulation by the Fur Protein. J Bacteriol 181:6223-6229.

Fillat MF. 2014. The FUR (ferric uptake regulator) superfamily: Diversity and versatility of key transcriptional regulators. Arch Biochem Biophys 546:41-52.

Gorska A, Sloderbach A, and Marszall MP. 2014. Siderophore-drug complexes: potential medicinal applications of the 'Trojan horse' strategy. Trends Pharmacol Sci 35:442-449. 10.1016/j.tips.2014.06.007

Gottesman S. 2005. Micros for microbes: non-coding regulatory RNAs in bacteria. Trends Genet 21:399404.

Hantke K. 2001. Iron and metal regulation in bacteria. Curr Opin Microbiol 4:172 - 177.

Hassett DJ, Sokol PA, Howell ML, Ma JF, Schweizer HT, Ochsner U, and Vasil ML. 1996. Ferric uptake regulator (Fur) mutants of Pseudomonas aeruginosa demonstrate defective siderophoremediated iron uptake, altered aerobic growth, and decreased superoxide dismutase and catalase activities. J Bacteriol 178:3996-4003.

Hjerde E, Lorentzen M, Holden M, Seeger K, Paulsen S, and Bason N. 2008. The genome sequence of the fish pathogen Aliivibrio salmonicida strain LFI1238 shows extensive evidence of gene decay. BMC Genomics 9:616.

Jacques JF, Jang S, Prévost K, Desnoyers G, Desmarais M, Imlay J, Massé E. 2006. RyhB small RNA modulates the free intracellular iron pool and is essential for normal growth during iron limitation in Escherichia coli. Mol Microbiol 62(4):1181-90.

Hobbs EC, Fontaine F, Yin X, and Storz G. 2011. An expanding universe of small proteins. Curr Opin Microbiol 14:167-173.

Kadi N, Song L, and Challis G. 2008. Bisucaberin biosynthesis: an adenylating domain of the BibC multienzyme catalyzes cyclodimerization of N-hydroxy-N-succinylcadaverine. Chem Commun (Camb) 41:5119 - 5121.

Kery MB, Feldman M, Livny J, and Tjaden B. 2014. TargetRNA2: identifying targets of small regulatory RNAs in bacteria. Nucleic Acids Res 42:W124-W129.

Keseler IM, Mackie A, Peralta-Gil M, Santos-Zavaleta A, Gama-Castro S, Bonavides-Martínez C, Fulcher C, Huerta AM, Kothari A, Krummenacker M, Latendresse M, Muñiz-Rascado L, Ong Q, Paley S, 
600

601

602

603

604

605

606

607

608

609

610

611

612

613

614

615

616

617

618

619

620

621

622

623

624

625

626

627

628

629

630

631

632

633

634

635

636

637

638

639

640

641

642

643

644

645

646

Schröder I, Shearer AG, Subhraveti P, Travers M, Weerasinghe D, Weiss V, Collado-Vides J, Gunsalus RP, Paulsen I, and Karp PD. 2013. EcoCyc: fusing model organism databases with systems biology. Nucleic Acids Res 41:D605-D612.

Masse E, Escorcia FE, and Gottesman S. 2003. Coupled degradation of a small regulatory RNA and its mRNA targets in Escherichia coli. Genes Dev 17:2374-2383.

Massé E, and Gottesman S. 2002. A small RNA regulates the expression of genes involved in iron metabolism in Escherichia coli. P Nat Acad Sci 99:4620-4625.

Masse E, Vanderpool C, and Gottesman S. 2005. Effect of RyhB small RNA on global iron use in Escherichia coli. J Bacteriol 187:6962 - 6971.

McClure R, Balasubramanian D, Sun Y, Bobrovskyy M, Sumby P, Genco CA, Vanderpool CK, and Tjaden B. 2013. Computational analysis of bacterial RNA-Seq data. Nucleic Acids Res 41:e140.

McHugh J, Rodriguez-Quinones F, Abdul-Tehrani H, Svistunenko D, Poole R, and Cooper C. 2003. Global iron-dependent gene regulation in Escherichia coli. J Biological Chem 278:29478 - 29486.

Mey A, Wyckoff E, Kanukurthy V, Fisher C, and Payne S. 2005a. Iron and Fur regulation in Vibrio cholerae and the role of Fur in virulence. Infect Immun 73:8167 - 8178.

Mey AR, Craig SA, and Payne SM. 2005b. Characterization of Vibrio cholerae RyhB: the RyhB Regulon and Role of ryhB in Biofilm Formation. Infect Immun 73:5706-5719.

Miller RE, Stadtman ER. 1972. Glutamate synthase from Escherichia coli. An iron-sulfide flavoprotein. J Biol Chem 247(22):7407-19.

Milton DL, O'Toole R, Horstedt P, and Wolf-Watz H. 1996. Flagellin A is essential for the virulence of Vibrio anguillarum. $J$ Bacteriol 178.

Murphy ER, and Payne SM. 2007. RyhB, an Iron-Responsive Small RNA Molecule, Regulates Shigella dysenteriae Virulence. Infect Immun 75:3470-3477.

Oglesby-Sherrouse AG, and Murphy ER. 2013. Iron-Responsive Bacterial Small RNAs: Variations on a Theme. Metallomics 5:276-286.

Pajuelo D, Hernandez-Cabanyero C, Sanjuan E, Lee CT, Silva-Hernandez FX, Hor LI, MacKenzie S, and Amaro C. 2016. Iron and Fur in the life cycle of the zoonotic pathogen Vibrio vulnificus. Environ Microbiol.

Pecqueur L, D'Autreaux B, Dupuy J, Nicolet Y, Jacquamet L, Brutscher B, Michaud-Soret I, and Bersch B. 2006. Structural changes of Escherichia coli ferric uptake regulator during metal-dependent dimerization and activation explored by NMR and X-ray crystallography. J Biol Chem 281:2128621295.

Pedersen HL, Ahmad R, Riise EK, Leiros HK, Hauglid S, Espelid S, Brandsdal BO, Leiros I, Willassen NP, and Haugen P. 2010. Experimental and computational characterization of the ferric uptake regulator from Aliivibrio salmonicida (Vibrio salmonicida). J Microbiol 48:174-183.

Pohl E, Haller JC, Mijovilovich A, Meyer-Klaucke W, Garman E, and Vasil ML. 2003. Architecture of a protein central to iron homeostasis: crystal structure and spectroscopic analysis of the ferric uptake regulator. Mol Microbiol 47:903-915.

Saha R, Saha N, Donofrio RS, and Bestervelt LL. 2013. Microbial siderophores: a mini review. J Basic Microbiol 53:303-317.

Seo SW, Kim D, Latif H, O'Brien EJ, Szubin R, Palsson BO. 2014. Deciphering Fur transcriptional regulatory network highlights its complex role beyond iron metabolism in Escherichia coli. Nat Commun 5:4910

Serres MH, Riley M. 2000. MultiFun, a multifunctional classification scheme for Escherichia coli K-12 gene products. Microb Comp Genomics. 5:205-222.

Sheikh M, and Taylor G. 2009. Crystal structure of the Vibrio cholerae ferric uptake regulator (Fur) reveals insights into metal co-ordination. Mol Microbiol 72:1208 - 1220. 
647 648 649
Smaldone GT, Revelles O, Gaballa A, Sauer U, Antelmann H, Helmann JD. 2012. A global investigation of the Bacillus subtilis iron-sparing response identifies major changes in metabolism. J Bacteriol 194(10):2594-605.

Thode SK, Kahlke T, Robertsen EM, Hansen H, and Haugen P. 2015. The immediate global responses of Aliivibrio salmonicida to iron limitations. BMC Microbiol 15:1-9.

Toffano-Nioche C, Nguyen AN, Kuchly C, Ott A, Gautheret D, Bouloc P, and Jacq A. 2012. Transcriptomic profiling of the oyster pathogen Vibrio splendidus opens a window on the evolutionary dynamics of the small RNA repertoire in the Vibrio genus. RNA 18:2201-2219.

Touati D. 2000. Iron and oxidative stress in bacteria. Arch of Biochem Biophys 373:1 - 6 .

van der Meulen SB, de Jong A, and Kok J. 2016. Transcriptome landscape of Lactococcus lactis reveals many novel RNAs including a small regulatory RNA involved in carbon uptake and metabolism. RNA Biol 13:353-366.

Waterhouse AM, Procter JB, Martin DMA, Clamp M and Barton GJ. 2009. Jalview Version 2 - a multiple sequence alignment editor and analysis workbench. Bioinformatics 25(9): 1189-1191

Yang X-W, He Y, Xu J, Xiao X, and Wang F-P. 2013. The Regulatory Role of Ferric Uptake Regulator (Fur) during Anaerobic Respiration of Shewanella piezotolerans WP3. PLOS ONE 8:e75588. 


\section{Figure 1}

Functional distribution of genes that are $\geq 2 \times$ differentially expressed between $A$. salmonicida wild-type and a fur null mutant strain.

Numbers in parentheses represent percentage of the total number of genes within the genome in each functional class. For complete list of differentially expressed genes, see Table S2.

Not classified (included putative assignments) (4\%)

Regulation (5.9\%)

Extrachromosomal / foreign DNA (16.7\%)

Ribosome constituents (1.6\%)

Cell envelope (16\%)

Nucleotide biosynthesis $(0.7 \%)$

Fatty acid biosynthesis $(0.7 \%)$

Energy metabolism, carbon (3.4\%)

Degradation of small molecules (1.8\%)

Central intermediary metabolism (3.2\%)

Biosynthesis of cofactors, carriers (3.2\%)

Amino acid biosynthesis (2.1\%)

Macromolecule synthesis, modification (8.2\%)

Macromolecule metabolism (2.9\%)

Cell division (0.7\%)

Adaptation (0.7\%)

Transport/binding proteins (9.7\%)

Protection responses (1.1\%)

Cell processes $(2.8 \%)$

Unknown function, no known homologues (14.6\%)

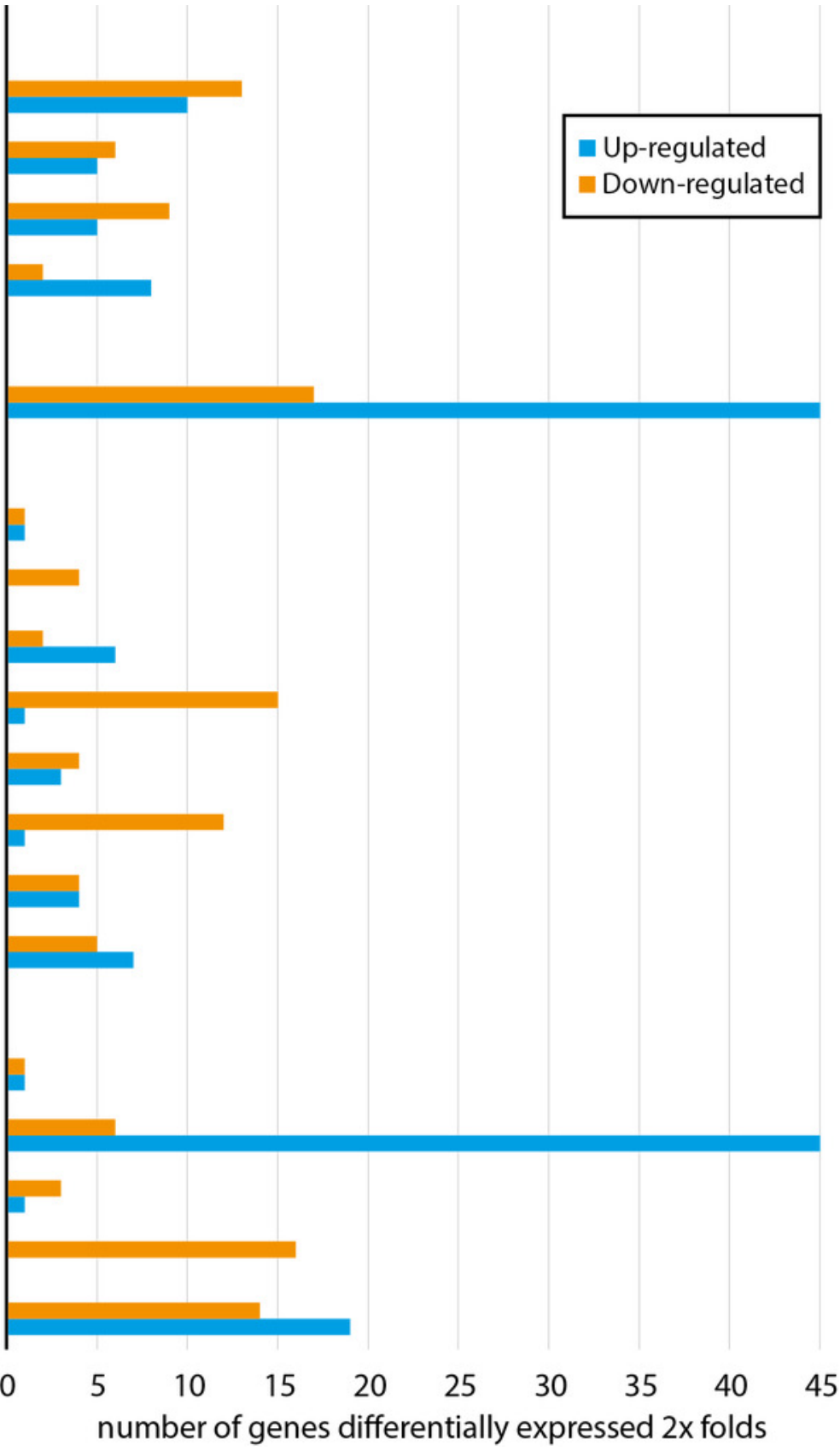


Figure 2

Schematic circular diagrams of the A. salmonicida chromosomes I (A) and II (B) (Chrl and Chrll).

The scale of the circles is in base-pairs. More than or equal to $4 \times$ differentially expressed genes are indicated with light blue filled circles and $\leq-3 \times$ differentially expressed genes are indicated with orange filled circles. Figure is not to scale.

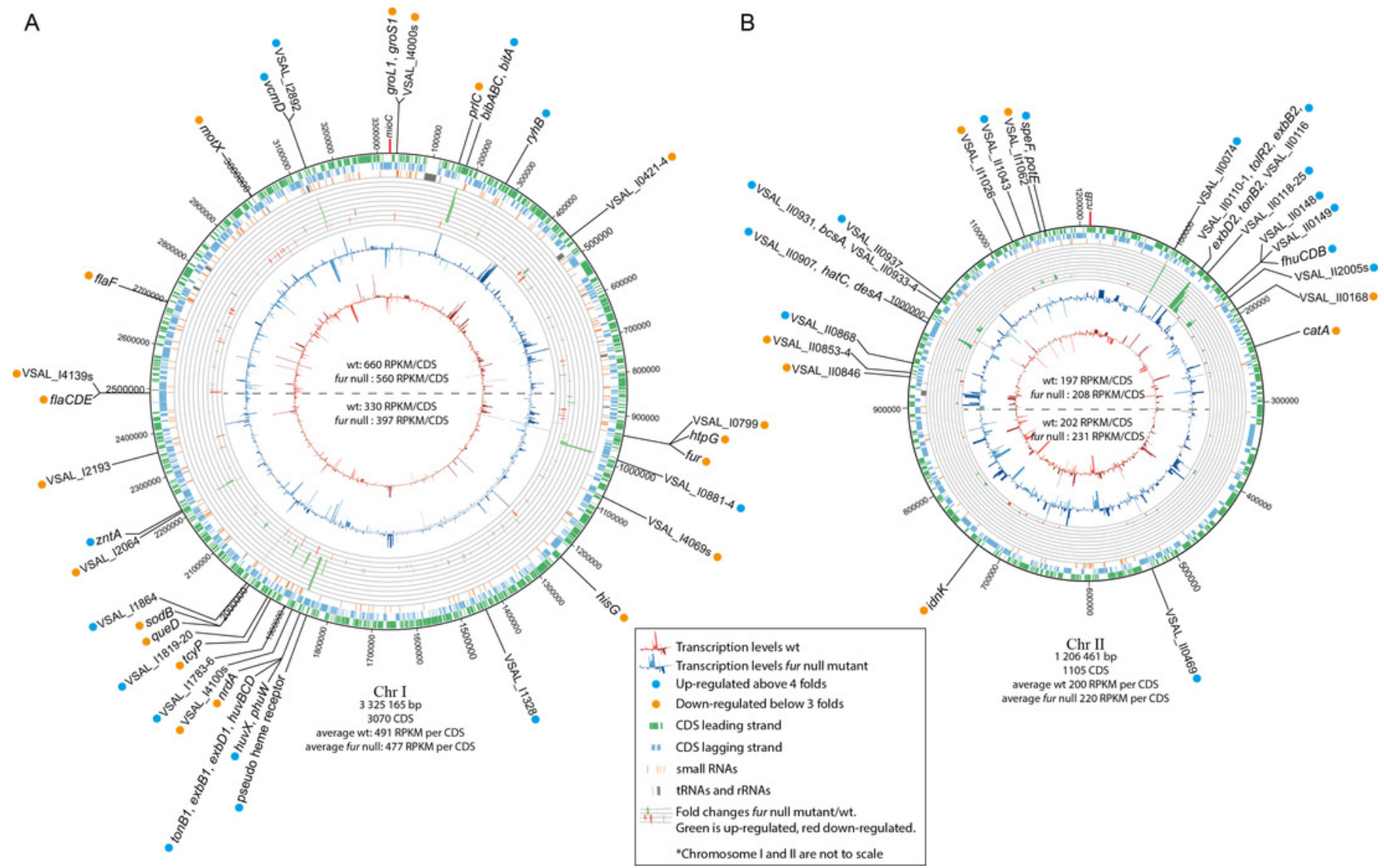




\section{Figure 3}

Relative expression levels for a selection of CDSs.

A) VSAL_10134-VSAL_10137; B) VSAL_II0110-VSAL_II0116; C) VSAL_I1751-VSAL_1756; D)

VSAL_13102s; E) VSAL_I10215; F) VSAL_12317-VSAL_I2319; G) VSAL_12771; H) VSAL_1858. Yaxis indicate the number of mapped reads. Red and blue curves represent mapped reads for wild-type and fur null mutant, respectively. The synteny of CDSs are shown below the graphs with associated numbers indicating the differential expression value ( $\Delta f u r / w t)$.

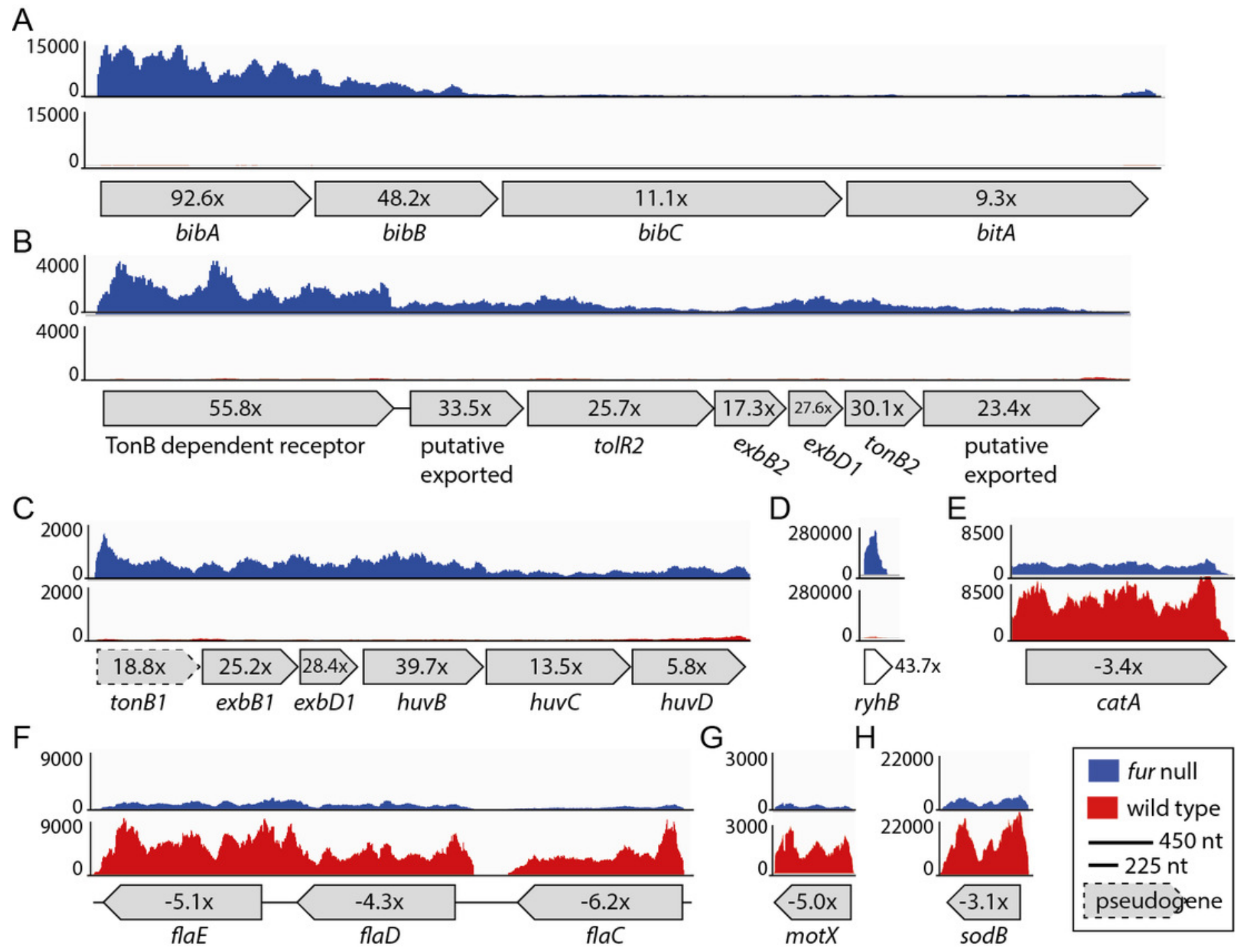




\section{Figure 4}

sRNAs identified by Rockhopper and manual curation.

A) sRNA 1 chromosome I position: 51134..51393. B) sRNA 2 chromosome I position:

776673..776837. C) sRNA 3 chromosome I position: 2343220..2343291. D) sRNA 4 chromosome I position: 2405357..2405638. E) sRNA 5 chromosome I position: 2812966..2813103. F) sRNA 6 chromosome I position: 3259173..3259344. G) sRNA 7 chromosome II position: 692443..692539. H) sRNA 8 chromosome II position: 814013..814056. I) SRNA 9 chromosome II position: 1141984..1142209. Y-axis indicate the number of mapped reads. Orange and turquoise curves represent mapped reads for wildtype and fur null mutant, respectively. sRNA genes are shown below curves, and associated numbers indicate the differential expression value ( $\Delta$ fur/wt). Small grey arrow heads indicate the distance in nt to flanking CDSs.
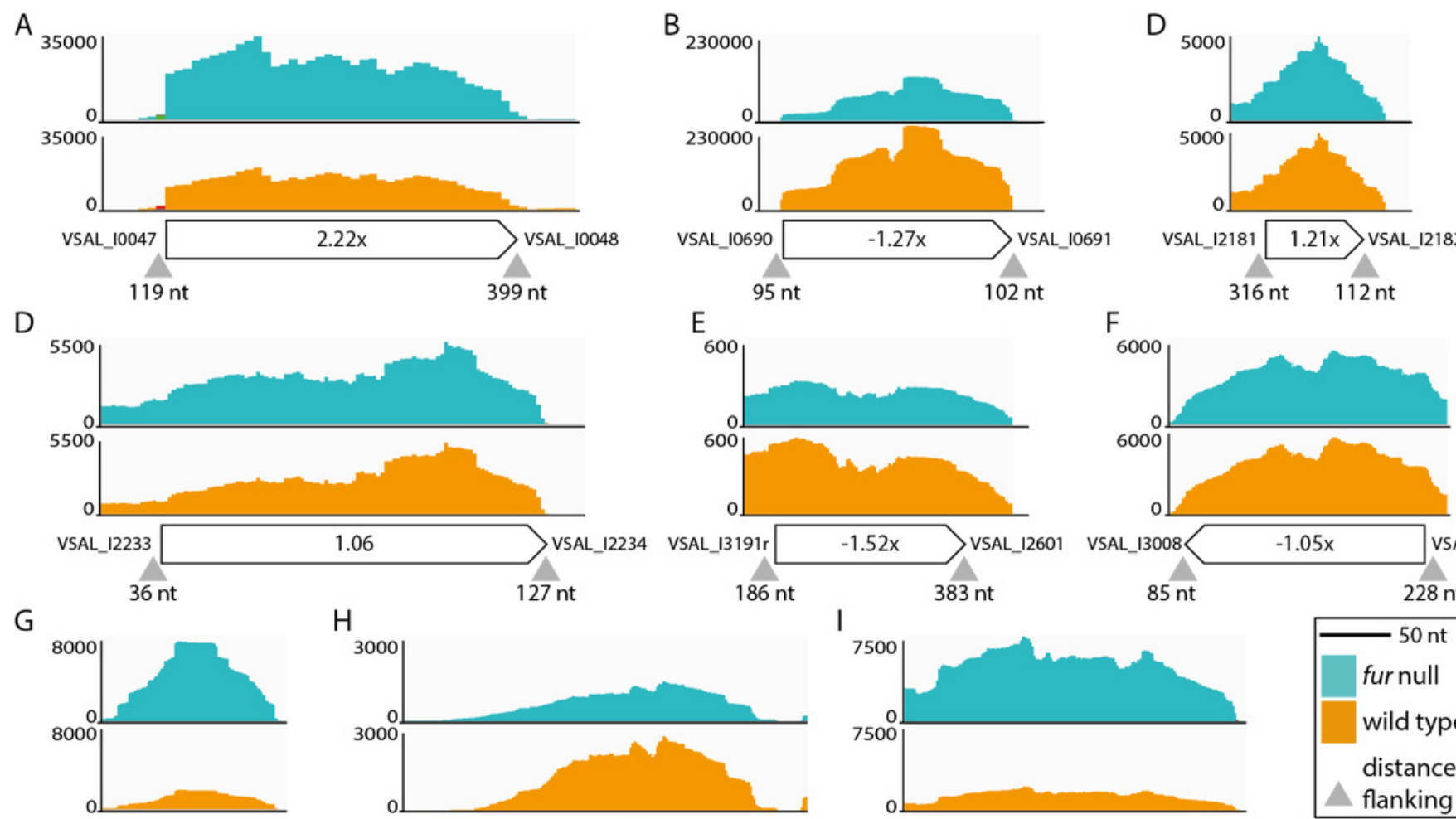

$\mathrm{H}$
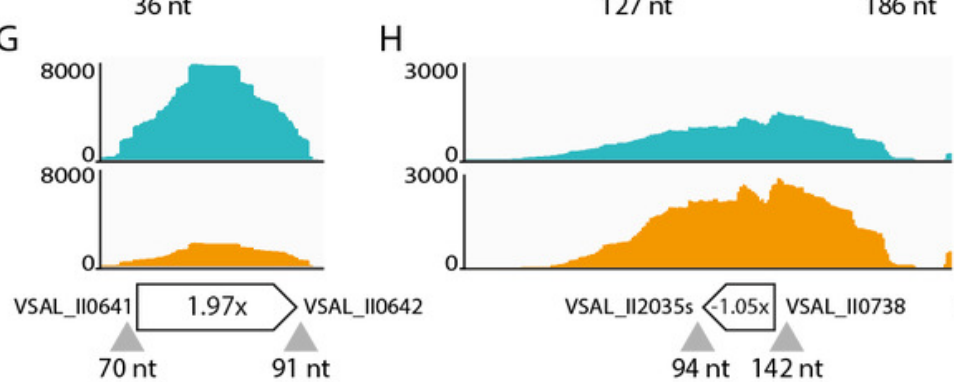

VSAL_II2035s $-1.05 \mathrm{x}$ VSAL_I10738

94 nt $142 \mathrm{nt}$ VSAL 11046

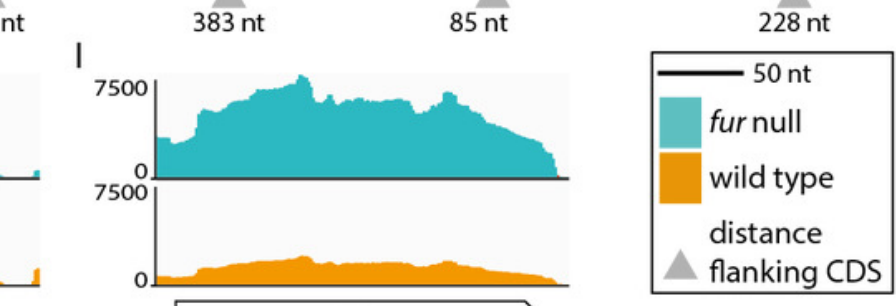
$-1.05 x$ VSAL_I3009

$70 \mathrm{nt} \quad 91 \mathrm{nt}$ 


\section{Table $\mathbf{1}$ (on next page)}

Up-regulated $(\geq 4 \times)$ genes in $A$. salmonicida fur null mutant compared to wild-type. 
Fur-

$\Delta$ furl box

\begin{tabular}{l} 
VSAL_nr gene Annotation \\
\hline Siderophore biosynthesis and transport
\end{tabular}

$\begin{array}{lllr}\text { VSAL_I0134 * } & \text { bibA } & \text { Bisucaberin siderophore biosynthesis protein A } & 92.6 \times \\ \text { VSAL_I0135 } & \text { bibB } & \text { Bisucaberin siderophore biosynthesis protein B } & 48.2 \times \\ \text { VSAL_I0136 } & \text { bibC } & \text { Bisucaberin siderophore biosynthesis protein C } & 11.1 \times \\ \text { VSAL_I0137 } & \text { bitA } & \text { TonB-dependent iron-siderophore receptor precursor } & 9.3 \times \\ \text { VSAL_II0148 } & & \text { 2Fe-2S binding protein, siderophore ferric reductase } & 8.0 \times \\ \text { VSAL_II0150 } & \text { fhuC } & \text { ferrichrome transport ATP-binding protein FhuC } & 7.0 \times \\ \text { VSAL_II0151 } & \text { fhuD } & \text { ferrichrome-binding periplasmic protein } & 12.5 \times \\ \text { VSAL_II0152 } & \text { fhuB } & \text { ferrichrome transport protein FhuB } & 6.7 \times \\ \text { VSAL_II0907 } & & \text { iron(III) ABC transporter, periplasmic iron-compound- } & 5.9 \times \\ \text { VSAL_II0908 } & \text { hatC } & \text { iron(III) ABC transporter, ATP-binding protein } & 11.2 \times \\ \text { VSAL_II0909 } & \text { desA } & \text { ferrioxamine B receptor } & 18.8 \times\end{array}$

TonB systems

tonB1 TonB protein (pseudogene)

$18.8 \times$

VSAL_I1752 exbB1 TonB system transport protein ExbB1

$25.2 \times$

VSAL_I1753

exbD1 TonB system transport protein ExbD1

$28.4 \times$

VSAL_II0110

TonB dependent receptor

$55.8 \times$

VSAL_II0111

putative exported protein

$35.3 \times$

VSAL_II0112

tolR2 biopolymer transport protein TolR

$25.7 \times$

VSAL_II0113

exbB2 TonB system transport protein ExbB2

$17.3 \times$

VSAL_II0114

exbD2 TonB system transport protein ExbD2

$27.6 x$

VSAL_II0115

tonB2 TonB protein

$30.1 \times$

VSAL_II0116

putative exported protein

$23.4 \times$

Heme uptake and utilization

VSAL_I1734

heme receptor (pseudogene)

$6.6 \times$

VSAL_I1749

huvX heme uptake and utilization protein HuvX

$20.2 \times$

VSAL_I1750

phuw

putative coproporphyrinogen oxidase PhuW

$39.7 \times$

VSAL_I1754 huvB protein

$39.7 \times$

VSAL_I1755 huvC

heme transporter protein HuvC, transmembrane

$39.7 \times$

$13.5 \times$

VSAL_I1756 heme transporter protein HuvD, ATP-binding

SMaIl RNA

VSAL_I3102s ryhB

small RNA RyhB

$43.7 \times$

VSAL_II2005s

VSAsRNA006

4.0

Other transport

VSAL_I1819

outer membrane protein $\mathrm{A}$ 


\begin{tabular}{|c|c|c|c|c|}
\hline VSAL_I2067 & $z n t A$ & lead, cadmium, zinc and mercury-transporting ATPase & 8.5 & \\
\hline VSAL_I2891 & vcmD & multidrug efflux pump & 8.5 & $x$ \\
\hline VSAL_II0118 & & membrane protein & 16.9 & \\
\hline VSAL_II0119 & & putative exported protein & 25.7 & \\
\hline VSAL_II0120 & & nickel transporter & 16.7 & \\
\hline VSAL_II0121 & & putative exported protein & 16.7 & \\
\hline VSAL_II0122 & & $\begin{array}{l}\text { putative membrane protein } \\
\text { zinc } A B C \text { transporter periplasmic substrate binding }\end{array}$ & 8.7 & \\
\hline VSAL_II0123 & & protein & 7.4 & \\
\hline VSAL_II0124 & & zinc $A B C$ transporter ATP binding protein & 6.3 & \\
\hline VSAL_II0125 & & zinc $A B C$ transporter permease & 4.1 & \\
\hline VSAL_II0149 & & $\begin{array}{l}\text { MFS transporter } \\
\text { cation efflux pump, cobalt-zinc-cadmium resistance }\end{array}$ & 5.6 & \\
\hline VSAL_II1043 & & protein & 5.7 & \\
\hline VSAL_II1067 & potE & putrescine-ornithine antiporter & 5.0 & \\
\hline \multicolumn{5}{|l|}{ Metabolism } \\
\hline VSAL_I1785 & & thiol oxioreductase & 5.7 & \\
\hline VSAL_I1786 & & peptidase, putative iron-regulated & 8.2 & $x$ \\
\hline VSAL_I2892 & & methyltransferase & 12.4 & $x$ \\
\hline VSAL_II0932 & $b c s A$ & cellulose synthase catalytic subunit & 6.1 & \\
\hline VSAL_II1066 & speF & ornithine decarboxylase, inducible & 7.4 & \\
\hline \multicolumn{5}{|l|}{ Cell envelope } \\
\hline VSAL_I1328 & & putative membrane associated peptidase & 4.4 & \\
\hline VSAL_I1783 & & putative lipoprotein & 4.4 & \\
\hline VSAL_I1784 & & putative lipoprotein & 5.0 & \\
\hline VSAL_I1820 & & putative lipoprotein & 4.0 & \\
\hline VSAL_I1864 & & putative membrane protein & 20.1 & $x$ \\
\hline VSAL_II0074 & & membrane protein & 67.3 & $x$ \\
\hline VSAL_II0868 & & putative lipoprotein & 8.0 & $x$ \\
\hline VSAL_II0931 & & membrane protein (fragment) & 4.8 & \\
\hline VSAL_II0933 & & putative exported protein & 6.2 & \\
\hline VSAL_II0937 & & membrane protein & 4.0 & \\
\hline \multicolumn{5}{|l|}{$\begin{array}{l}\text { Unknown } \\
\text { function }\end{array}$} \\
\hline VSAL_I0881 & & putative exported protein & 15.7 & $x$ \\
\hline VSAL_10882 & & putative exported protein & 14.1 & $x$ \\
\hline VSAL_I0883 & & putative exported protein & 14.4 & $x$ \\
\hline VSAL_I0884 & & putative exported protein & 5.0 & $x$ \\
\hline VSAL_II0469 & & hypothetical protein & 4.5 & \\
\hline VSAL_II0934 & & hypothetical protein & 4.0 & \\
\hline
\end{tabular}

* p-value not analyzed 
** fur-box predictions from Ahmad et.al. (Ahmad et al. 2009)

1 


\section{Table 2 (on next page)}

Down-regulated $(\leq-3 x)$ genes in $A$. salmonicida fur null mutant compared to wild-type. 


\begin{tabular}{|c|c|c|c|c|}
\hline VSAL_nr & gene & annotation & $\Delta$ fur/wt & sRNA target \\
\hline \multicolumn{5}{|c|}{ Motility/chemotaxis } \\
\hline VSAL_10799 & & methyl-accepting chemotaxis protein & -3.5 & \\
\hline VSAL_I2193* & & methyl-accepting chemotaxis protein & -3.6 & \\
\hline VSAL_I2317 & flaE & flaggelin subunit E & -5.1 & \\
\hline VSAL_I2318 & flaD & flaggelin subunit $D$ & -4.3 & \\
\hline VSAL_I2319 & flac & flaggelin subunit C & -6.2 & \\
\hline VSAL_I2517 & flaF & flaggelin subunit $F$ & -3.9 & \\
\hline VSAL_I2771 & $\operatorname{mot} X$ & sodium-type polar flagellar protein MotX & -5.0 & \\
\hline \multicolumn{5}{|c|}{ Oxidative stress response } \\
\hline VSAL_I1858 & $\operatorname{sod} B$ & superoxide dismutase $[\mathrm{Fe}]$ & -3.1 & RyhB \\
\hline VSAL_II0215 & $\operatorname{catA}$ & catalase & -3.4 & \\
\hline \multicolumn{5}{|c|}{ Metabolism } \\
\hline VSAL_I0122 & prlC & oligopeptidase A & -3.2 & \\
\hline VSAL_I0421 & cysN & sulfate adenylyltransferase subunit 1 & -3.4 & RyhB \\
\hline VSAL_I0422 & & ion transporter superfamily protein & -3.8 & RyhB \\
\hline VSAL_I0423 & cysC & adenylylsulfate kinase & -4.0 & \\
\hline VSAL_I1133 & hisG & $\begin{array}{l}\text { ATP phosphoribosyltransferase } \\
\text { ribonucleoside-diphosphate reductase }\end{array}$ & -3.4 & \\
\hline VSAL_I1769 & $n r d A$ & alpha chain & -3.8 & \\
\hline VSAL_I1857 & queD & queuosine biosynthesis protein & -4.0 & \\
\hline VSAL_II0666 & $i d n K$ & thermosensitive gluconokinase & -4.4 & \\
\hline VSAL_II0846 & & putative acetyltransferase & -3.4 & \\
\hline VSAL_II1026 & & putative tryptophanyl-tRNA synthetase & -6.4 & RyhB \\
\hline \multicolumn{5}{|l|}{ small RNA } \\
\hline VSAL_14000s & & VSsRNA001 & -4.1 & \\
\hline VSAL_14069s & & VSsRNA070 & -3.4 & \\
\hline VSAL_I4100s & & VSsRNA 101 & -4.1 & \\
\hline VSAL_14139s & & VSsRNA140 & -3.9 & \\
\hline \multicolumn{5}{|c|}{ Chaperones/ heat shock proteins } \\
\hline VSAL_I0017 & groL1 & 60 kda chaperonin 1 & -3.2 & \\
\hline VSAL_I0018 & gros1 & $\begin{array}{l}10 \text { kDa chaperonin } 1 \\
\text { chaperone protein } \mathrm{HtpG} \text { (heat shock protein }\end{array}$ & -3.9 & \\
\hline VSAL_I0814 & htpG & $\mathrm{HtpG)}$ & -3.2 & \\
\hline \multicolumn{5}{|c|}{ Cell envelope/transport } \\
\hline VSAL_I1813 & $\operatorname{tcy} P$ & L-cystine transporter & -8.6 & $\begin{array}{l}\text { RyhB, } \\
\text { VSAL_II2005s }\end{array}$ \\
\hline VSAL_II0853 & & MFS transporter & -4.0 & \\
\hline VSAL_II0854 & & secretion protein, HlyD family & -3.9 & \\
\hline VSAL_II1062 & & membrane protein & -3.3 & \\
\hline
\end{tabular}

\section{Unknown function}




$\begin{array}{llll}\text { VSAL_I0424 } & \text { hypothetical protein } & -3.2 & \text { RyhB } \\ \text { VSAL_I2064 } & \text { conserved hypothetical protein } & -4.0 & \\ \text { VSAL_II0168 } & \text { putative exported protein } & -7.9 & \\ \text { Mutated gene/ control gene } & \end{array}$

VSAL_10833 fur ferric uptake regulator protein

$-128.7 \quad$ RyhB

*fur-box predicted in Ahmad et. al. (Ahmad et al. 2009) 